\title{
On limit cycles bifurcating from the infinity in discontinuous piecewise linear differential systems
}

\author{
Márcio R.A. Gouveia a , Jaume Llibre ${ }^{\mathrm{b}}$, Douglas D. Novaes ${ }^{\mathrm{c}, *}$ \\ a Departamento de Matemática, IBILCE-UNESP, Rua C. Colombo, 2265, CEP 15054-000 S. J. Rio Preto, São Paulo, Brazil \\ b Departament de Matemàtiques, Universitat Autònoma de Barcelona, 08193 Bellaterra, Barcelona, Catalonia, Spain \\ ' Departamento de Matemática, Universidade Estadual de Campinas, Rua Sérgio Baruque de Holanda, 651, Cidade Universitária Zeferino Vaz, \\ CEP 13083-859, Campinas, São Paulo, Brazil
}

\section{A R T I C L E I N F O}

\section{MSC:}

$34 \mathrm{~A} 36$

$34 \mathrm{C} 29$

$37 \mathrm{G} 15$

\section{Keywords:}

Discontinuous differential system

Piecewise linear differential system

Infinite periodic orbit

Limit cycle

Displacement function

\begin{abstract}
A B S T R A C T
In this paper we consider the linear differential center $(\dot{x}, \dot{y})=(-y, x)$ perturbed inside the class of all discontinuous piecewise linear differential systems with two zones separated by the straight line $y=0$. Using the Bendixson transformation we provide sufficient conditions to ensure the existence of a crossing limit cycle coming purely from the infinity. We also study the displacement function for a class of discontinuous piecewise smooth differential system.
\end{abstract}

c 2015 Elsevier Inc. All rights reserved.

\section{Introduction and statement of the main result}

Establishing sufficient conditions for the existence of periodic solutions, specially limit cycles, i.e. periodic solutions isolated in the set of all periodic solutions, is one of the main problems of the qualitative theory of planar differential systems. A classical way to produce and to study limit cycles is perturbing the periodic solutions of a center, which is a point having a neighborhood, except itself, filled by periodic solutions. This problem for smooth differential systems in the plane has been studied intensively, either for limit cycles bifurcating from finite periodic solutions (see, for instance, hundreds of references in the book [4]), or for limit cycles bifurcating from the infinity (see, for instance, [7,21,22]). In [8] the authors studied the bifurcation of a limit cycle from the infinity for a non-smooth but continuous piecewise differential system. Nevertheless, as far as we know, there are no studies of limit cycles bifurcating from the infinity for discontinuous differential systems. Accordingly, this is the objective of the present paper: to study limit cycles coming from the infinity for a class of discontinuous differential systems.

Recently the theory of discontinuous differential systems has been strongly developed, with growing importance at the frontier between mathematics, physics, engineering, and the life sciences. Interest stems particularly from discontinuous dynamical models (see, for instance, $[15,20]$ ). Additionally, the existence of periodic solutions gives important information about the dynamics of these models, as the presence of oscillatory motions (see, for instance, the book of Andronov, Vitt, and Khaikin [2], and the book of Minorski [16], both classical and important works on physical oscillatory phenomena), which represents one of the main source of physical motivation for this study. There are some works dealing with limit cycles bifurcating from finite periodic solutions in discontinuous differential systems, see, for instance, [3,9,10,12-14,17,18]. To the best of our knowledge, this paper is the first work dealing with limit cycles bifurcating from the infinity for discontinuous systems.

\footnotetext{
* Corresponding author. Tel.: +55 1935215921; fax: +55 1935216094 .

E-mail addresses: maralves@ibilce.unesp.br (M.R.A. Gouveia), jllibre@mat.uab.cat (J. Llibre), ddnovaes@ime.unicamp.br (D.D. Novaes).
} 


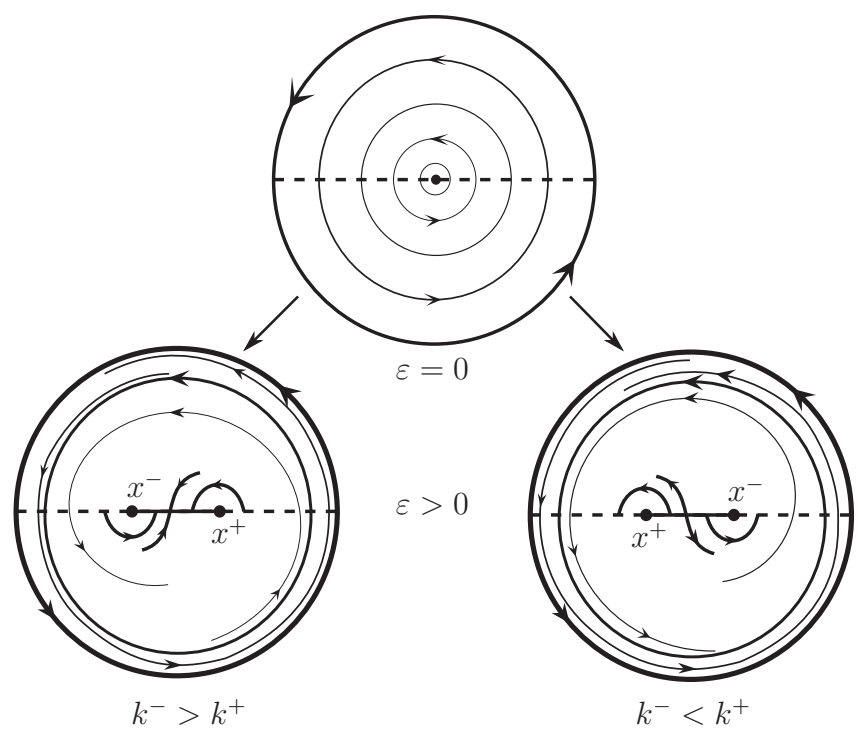

Fig. 1. Behavior of the periodic orbits (in the Poincaré disc) of system (1) satisfying the hypotheses (iii) of Theorem 1 . For $\varepsilon=0$ system (1) is a center. For $\varepsilon>0$ and $k^{-}>k^{+}$system (1) has an unstable limit cycle, near to the infinity, and a sliding region in the discontinuous set. For $\varepsilon>0$ and $k^{-}<k^{+}$system (1) has a stable limit cycle, near to the infinity, and a scape region in the discontinuous set. The diameters crossing the discs represents the discontinuous set $\Sigma$, where the dashed lines are the crossing regions $\Sigma^{c}$ and the continuous lines are the sliding $\Sigma^{s}$ and escaping $\Sigma^{e}$ regions when $k^{-}>k^{+}$and $k^{-}<k^{+}$, respectively. The points $\left(x^{ \pm}, 0\right)$ are called invisible folds of the vector fields $F^{ \pm}$. For a definition of Poincaré disc, see, for instance, Chapter 5 of [5].

\subsection{Limit cycles from the infinity}

In this paper we consider the linear differential center $(\dot{x}, \dot{y})=(-y, x)$ perturbed inside the class of all discontinuous piecewise linear differential systems with two zones separated by the straight line $\Sigma=\{y=0\}$, that is

$$
\begin{gathered}
\dot{x}= \begin{cases}-y+\varepsilon\left(a^{+}+b^{+} x+c^{+} y\right)+\varepsilon^{2}\left(\alpha^{+}+\beta^{+} x+\gamma^{+} y\right) & \text { if } y>0, \\
-y+\varepsilon\left(a^{-}+b^{-} x+c^{-} y\right)+\varepsilon^{2}\left(\alpha^{-}+\beta^{-} x+\gamma^{-} y\right) & \text { if } y<0,\end{cases} \\
\dot{y}= \begin{cases}x+\varepsilon\left(k^{+}+m^{+} x+n^{+} y\right)+\varepsilon^{2}\left(\kappa^{+}+\mu^{+} x+v^{+} y\right) & \text { if } y>0, \\
x+\varepsilon\left(k^{-}+m^{-} x+n^{-} y\right)+\varepsilon^{2}\left(\kappa^{-}+\mu^{-} x+v^{-} y\right) & \text { if } y<0 .\end{cases}
\end{gathered}
$$

The dot denotes derivative with respect to the time $t$.

Here we are interested in the study of crossing limit cycles, that is limit cycles that do not contain sliding segments and tangential points. Roughly speaking, we say that a periodic solution $(x(t, \varepsilon), y(t, \varepsilon))$ comes purely from the infinity if $\|(x(t, \varepsilon)$, $y(t, \varepsilon)) \| \rightarrow \infty$ when $\varepsilon \rightarrow 0$ for every $t \in \mathbb{R}$. In [3] the authors found, for a class of discontinuous piecewise linear perturbation of the linear center, conditions for the existence of periodic solutions $(x(t, \varepsilon), y(t, \varepsilon))$ such that $\|(x(t, \varepsilon), y(t, \varepsilon))\| \rightarrow \lambda$ when $\varepsilon \rightarrow 0$, moreover the level $\lambda \in \mathbb{R}$ can be taken as bigger as wanted. Nevertheless, since $\|(x(t, \varepsilon), y(t, \varepsilon))\|=\lambda+\mathcal{O}(\varepsilon)$, taking $\lambda \rightarrow \infty$ is not sufficient to prove the existence of a periodic solution coming purely from the infinity for some perturbation. Our first main result in this paper is about the crossing limit cycles of system (1). For completeness, in the statements (i) and (ii) we study the birth (and the uniqueness) of a crossing limit cycle that bifurcates from the origin and from the periodic solutions of the linear center (that is, system (1) when $\varepsilon=0$ ), respectively. The crossing limit cycle bifurcating purely from the infinity is studied in the statement (iii).

Theorem 1. Regarding to system (1) the following statements hold.

(i) If $\left(b^{+}+b^{-}+n^{+}+n^{-}\right)\left(k^{-}-k^{+}\right)>0$ then, for $|\varepsilon| \neq 0$ sufficiently small, there exists a unique crossing limit cycle $\left(x_{1}(t\right.$, $\varepsilon)$, $y_{1}(t, \varepsilon)$ ) of system (1) which is stable (resp. unstable) provided that $b^{+}+b^{-}+n^{+}+n^{-}<0$ (resp. $\left.b^{+}+b^{-}+n^{+}+n^{-}>0\right)$. Moreover $\left\|\left(x_{1}(t, \varepsilon), y_{1}(t, \varepsilon)\right)\right\| \rightarrow 4\left(k^{-}-k^{+}\right) /\left(\pi\left(b^{+}+b^{-}+n^{+}+n^{-}\right)\right)>0$ when $\varepsilon \rightarrow 0$ for every $t \in \mathbb{R}$.

(ii) If $k^{-}-k^{+}=0$ and $\left(b^{+}+b^{-}+n^{+}+n^{-}\right)\left(4 a^{-}\left(b^{-}+n^{-}\right)-4\left(k^{+}\left(m^{-}-m^{+}\right)+a^{+}\left(b^{+}+n^{+}\right)+\kappa^{+}-\kappa^{-}\right)-k^{+}\left(b^{+}+b^{-}+n^{+}+\right.\right.$ $\left.\left.n^{-}\right)\right)>0$ then, for $\varepsilon>0$ sufficiently small, there exists a unique crossing limit cycle $\left(x_{2}(t, \varepsilon), y_{2}(t, \varepsilon)\right)$ of system (1) which is stable (resp. unstable) provided that $b^{+}+b^{-}+n^{+}+n^{-}<0$ (resp. $\left.b^{+}+b^{-}+n^{+}+n^{-}>0\right)$. Moreover $\left\|\left(x_{2}(t, \varepsilon), y_{2}(t, \varepsilon)\right)\right\| \rightarrow$ 0 when $\varepsilon \rightarrow 0$ for every $t \in \mathbb{R}$.

(iii) If $b^{+}+b^{-}+n^{+}+n^{-}=0$ and $\left(k^{-}-k^{+}\right)\left(\left(b^{+}+n^{+}\right)\left(c^{-}-c^{+}+m^{+}-m^{-}\right)-2\left(\beta^{+}+\beta^{+}+v^{+}+v^{-}\right)\right)<0$ then, for $\varepsilon>0$ sufficiently small, there exists a unique crossing limit cycle $\left(x_{3}(t, \varepsilon), y_{3}(t, \varepsilon)\right)$ of system (1) which is stable (resp. unstable) provided that $k^{-}<k^{+}$(resp. $k^{-}>k^{+}$). Moreover this limit cycle comes purely from the infinity, that is $\left\|\left(x_{3}(t, \varepsilon), y_{3}(t, \varepsilon)\right)\right\| \rightarrow \infty$ when $\varepsilon \rightarrow 0$ for every $t \in \mathbb{R}$ (see Fig. 1 ). 

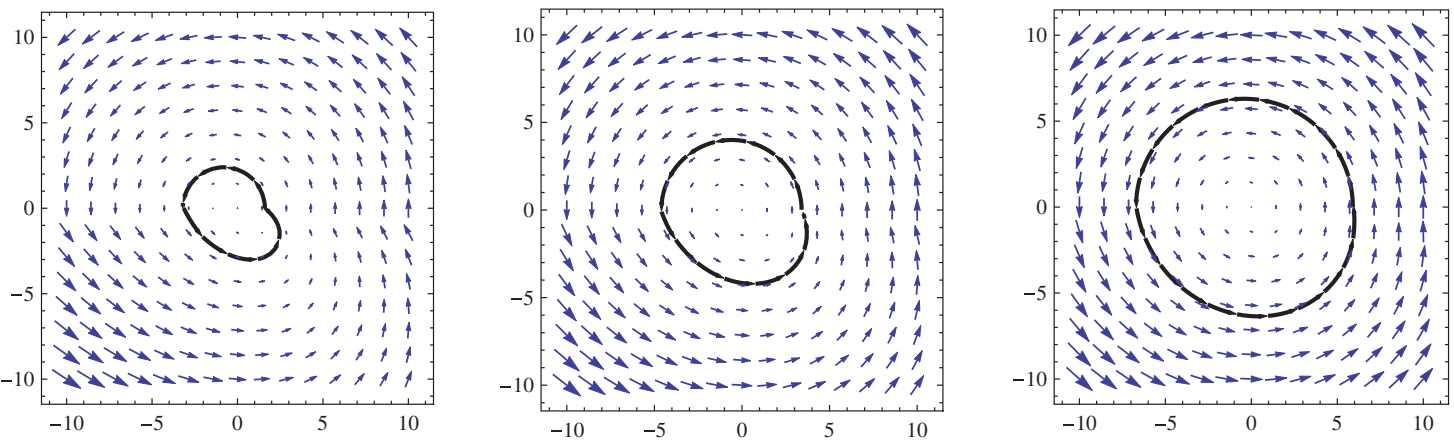

Fig. 2. Limit cycle of $(\dot{x}, \dot{y})=(-y, x+\varepsilon)$ for $y>0$, and $(\dot{x}, \dot{y})=\left(-y-\varepsilon^{2} x, x-\varepsilon\right)$ for $y<0$. The variable $\varepsilon$ are assuming the values $0.8,0.6$ and 0.4 , respectively. We observe that the amplitude of the periodic orbit becomes bigger when $\varepsilon$ goes to 0 .

Theorem 1 is proved in Section 3.

We note that the parameter $\delta_{1}=k^{-}-k^{+}$controls whether the limit cycle comes from the origin or not, and the parameter $\delta_{2}=b^{+}+b^{-}+n^{+}+n^{-}$controls whether the limit cycle comes from the infinity or not. In other words, Theorem 1 shows us that, assuming some conditions, the amplitude of the limit cycle given by statement $(i)$ increases and decreases continuously over the plane always when we fix one of the parameters, $\delta_{1}$ or $\delta_{2}$, and make the other one tending to zero.

System (1) belongs to a bigger class of planar piecewise differential systems, $(\dot{x}, \dot{y})=F^{+}(x, y)$ if $h(x, y)>0$, and $(\dot{x}, \dot{y})=$ $F^{-}(x, y)$ if $h(x, y)<0$, where $\Sigma=h^{-1}(0)$ is a regular manifold and $F^{ \pm}$are planar smooth vector fields (in our case $h(x, y)=y$ ). It is well known that $\Sigma$ can be generically decompose in three regions, namely, (crossing region) $\Sigma^{c}=\{x \in \Sigma$ : $\left.\left(F^{+} h\right)\left(F^{-} h\right)(x, y)>0\right\}$, (escaping region) $\Sigma^{e}=\left\{x \in \Sigma:\left(F^{+} h\right)(x, y)>0\right.$ and $\left.\left(F^{-} h\right)(x, y)<0\right\}$, and (sliding region) $\Sigma^{s}=\{x \in \Sigma$ : $\left(F^{+} h\right)(x, y)<0$ and $\left.\left(F^{-} h\right)(x, y)>0\right\}$, that is $\Sigma=\overline{\Sigma^{c}} \cup \overline{\Sigma^{e}} \cup \overline{\Sigma^{s}}$. As usual, $\left(F^{+} h\right)(x, y)=\left\langle\nabla h(x, y), F^{+}(x, y)\right\rangle$ where $\langle\cdot, \cdot\rangle$ denotes the dot product of $\mathbb{R}^{2}$. A local solution, starting at a point $p \in \Sigma$, of a piecewise differential system is given by the Filippov's convention (see [6]). The most important thing to know, in this paper, is that the solution passing through a point $p \in \Sigma^{c}$ is just the concatenation of the solutions arriving by one side and leaving by the other side.

Let

$$
\begin{aligned}
& x^{+}=\frac{-\varepsilon k^{+}-\varepsilon^{2} \kappa^{+}}{1+\varepsilon m^{+}+\varepsilon^{2} \mu^{+}}=-\varepsilon k^{+}+\mathcal{O}\left(\varepsilon^{2}\right) \quad \text { and } \\
& x^{-}=\frac{-\varepsilon k^{-}-\varepsilon^{2} \kappa^{-}}{1+\varepsilon m^{-}+\varepsilon^{2} \mu^{-}}=-\varepsilon k^{-}+\mathcal{O}\left(\varepsilon^{2}\right) .
\end{aligned}
$$

Clearly, under the hypotheses of the statement (i) or (iii) of Theorem $1, x^{+} \neq x^{-}$for $\varepsilon>0$ small enough. So, regarding system (1) we have: if $k^{-}<k^{+}$then $\Sigma^{e}=\left\{(x, 0): x^{+}<x<x^{-}\right\}, \Sigma^{s}=\emptyset$, and $\Sigma^{c}=\mathbb{R} \backslash \overline{\Sigma^{e}}$; and if $k^{-}>k^{+}$then $\Sigma^{e}=\emptyset, \Sigma^{s}=\left\{(x, 0): x^{-}<\right.$ $x<x^{+}$, and $\Sigma^{c}=\mathbb{R} \backslash \overline{\Sigma^{s}}$ (see Fig. 1). We note that the condition $k^{-}<k^{+}$(resp. $k^{-}>k^{+}$) of Theorem 1, about the stability of the limit cycle, is the same which controls the existence of an escaping (resp. sliding) region. The escape (resp. sliding) region creates an unstable (resp. stable) region near to $\Sigma^{e}$ (resp. $\Sigma^{S}$ ), which agrees with the stability of the limit cycle predicted by Theorem 1 . Nevertheless it does not imply that the orbits leaving (resp. arriving to) the escape (resp. sliding) region have to accumulate in (resp. come from) this limit cycle. This fact will be clear in the next example.

Example 1. Taking $(\dot{x}, \dot{y})=(-y, x+\varepsilon)$ when $y>0$, and $(\dot{x}, \dot{y})=\left(-y-\varepsilon^{2} x, x-\varepsilon\right)$ when $y<0$ we have a simple example of a discontinuous piecewise linear differential system for which the hypotheses of statement (iii) of Theorem 1 hold (see Figs. 2 and 3).

For completeness, in what follows, we shall describe the global phase portrait of this system for $\varepsilon=0.8$ in the Poincare disc (see Fig. 4). To do that we have used the software P5 which allows the study of piecewise planar polynomial phase portrait. The system has two equilibrium points, namely: $(-0.8,0)$ which is local repelling (even in the linear system defined for $y>0$ it is a center), and the point $(0.8,-0.5129)$ which is a stable focus. Furthermore, there are others two distinguished points, the origin $(0,0)$ which is a pseudo-equilibrium (an equilibrium of the sliding vector field), and the point $(0.8,0)$ which is a visible fold for the system restricted to $y<0$ (and the extreme of the sliding segment). The importance of these four points will be clear in the sequel.

The infinity (in the Poincare disc) is an unstable periodic orbit $U$, and $S$ is a stable limit cycle which bifurcates from the infinity for $\epsilon=0$. Inside the region delimited by $S$ there exists an unstable limit cycle $I$ that contains a sliding segment $[0.11,0.8] \times\{0\}$, approximately (we stress Theorem 1 does not detect such an orbit). The orbits into the region delimited by $S$ and $U$ converge to $S$ in forward time and converge to $U$ in backward time. Into the region delimited by the stable limit cycle $S$ and by the unstable limit cycle $I$ we have a similar behavior, but here the orbits converge to $S$ in forward time and converge to $I$ in backward time. The orbits passing through points inside the region delimited by $I$ but not in $[0.11,0.8] \times\{0\}$ converge to the stable focus $(0.8,-0.5129)$ in forward time, and reach the sliding segment $[0.11,0.8] \times\{0\}$ in a finite backward time. 


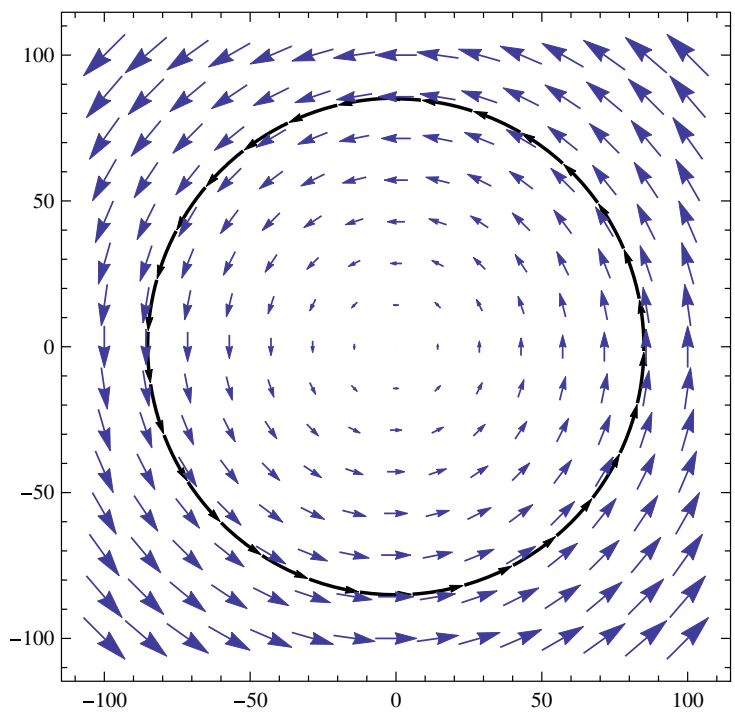

Fig. 3. The limit cycle for $\varepsilon=0.03$ of $(\dot{x}, \dot{y})=(-y, x+\varepsilon)$ for $y>0$, and $(\dot{x}, \dot{y})=\left(-y-\varepsilon^{2} x, x-\varepsilon\right)$ for $y<0$.

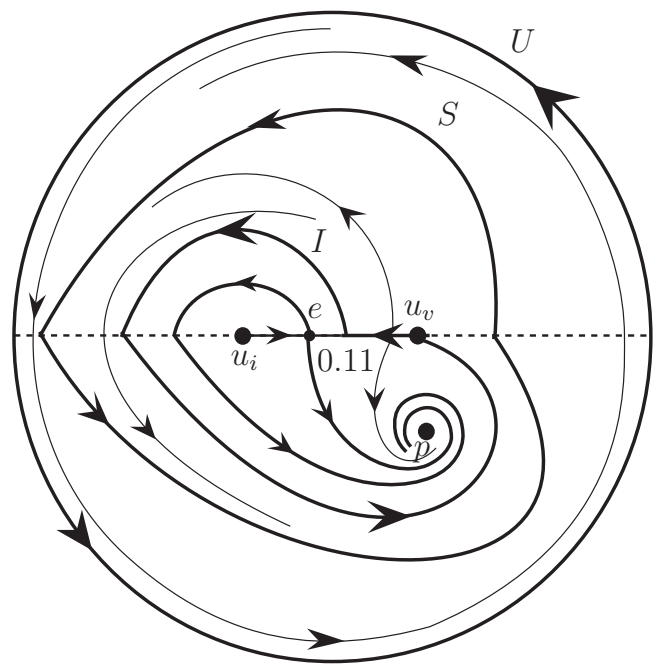

Fig. 4. Global phase portrait of system $(\dot{x}, \dot{y})=(-y, x+\varepsilon)$ for $y>0$, and $(\dot{x}, \dot{y})=\left(-y-\varepsilon^{2} x, x-\varepsilon\right)$, assuming $\varepsilon=0.8$. The infinity is an unstable periodic orbit $U$, and $S$ is a stable limit cycle which bifurcates from the infinity for $\epsilon=0$. Inside the region delimited by $S$ there exists an unstable limit cycle $I$ that contains a sliding segment $[0.11,0.8] \times\{0\}$. The orbits into the region delimited by $S$ and $U$ (resp. $S$ and $I$ ) converge to $S$ in forward time and converge to $U$ (resp. $I$ ) in backward time. The orbits passing through points inside the region delimited by $I$ but not in $\mathcal{L}=[0.11,0.8] \times\{0\}$ converge to the stable focus $p=(0.8,-0.5129)$ in forward time, and reach $\mathcal{L}$ in a finite backward time. Now for points $(x, 0) \in \mathcal{L}$ we have: (i) if $x \in[-0.8,0)$ then its orbit either converges to the pseudoequilibrium $e=(0,0)$ or converges to $p$ in forward time. In backward time its orbit reaches the invisible fold point $u_{i}=(-0.8,0)$; (ii) if $x=e$ then its orbit either remains stationary or converge to $p$ in forward time. In backward time its orbit remains stationary; (iii) if $x \in(0,0.11)$ then its orbit either converges to the pseudo equilibrium $e$ or converges to $p$ in forward time. In backward time its orbit reaches the visible fold $u_{v}=(0.8,0)$ and then remains on $I$; (iv) if $x \in[0.11,0.8]$ then its orbit either converges to the pseudo equilibrium $e$, or converges to $p$, or remains on $I$, or converges to $S$ in forward time. In backward time its orbit reaches the visible fold $u_{v}$ in a finite time and then remains on $I$.

Now we need to look more carefully to the points $(x, 0) \in[-0.8,0.8] \times\{0\}$ in order to understand the behaviour of the orbits passing through them. We shall have in mind the non-uniqueness property of an orbit passing through an escaping point $x \in \Sigma^{e}$ (resp. sliding point $x \in \Sigma^{S}$ ) in forward time (resp. backward time). We divide the sliding segment $[-0.8,0.8]$ as follows: $[-0.8,0.8]=[-0.8,0) \cup\{0\} \cup(0,0.11) \cup[0.11,0.8]$. So

(i) if $x \in[-0.8,0)$ then its orbit either converges to the pseudo equilibrium $(0,0)$ or converges to the stable focus $(0.8,-0.5129)$ in forward time. In backward time its orbit reaches the invisible fold point $(-0.8,0)$ in a finite time;

(ii) if $x=(0,0)$ then its orbit either remains stationary or converge to the stable focus $(0.8,-0.5129)$ in forward time. In backward time its orbit remains stationary; 


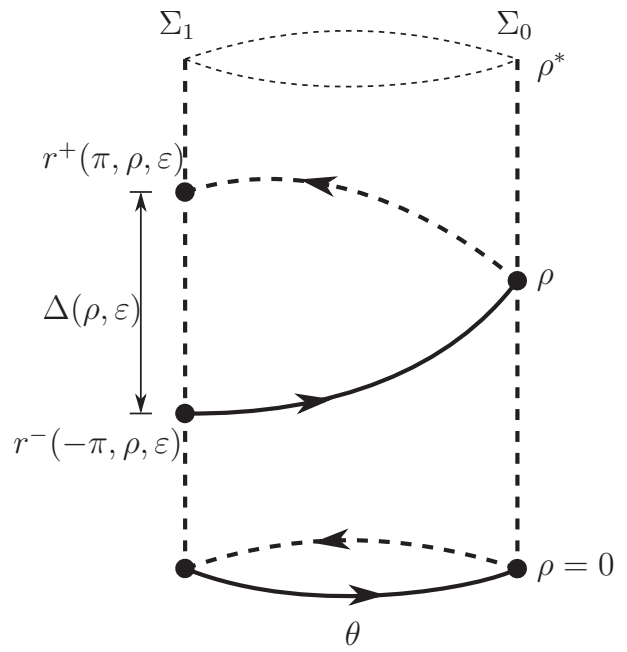

Fig. 5. For a fixed $\varepsilon>0$ the displacement function $\Delta$ related to system (2) evaluated at a point $\rho>0$ is defined as the difference between the position of the first return to $\Sigma_{1}$ in forward time and the position of the first return to $\Sigma_{1}$ in backward time, considering the flow passing through $\rho$.

(iii) if $x \in(0,0.11)$ then its orbit either converges to the pseudo equilibrium $(0,0)$ or converges to the stable focus $(0.8,-0.5129)$ in forward time. In backward time its orbit reaches the visible fold $(0.8,0)$ in a finite time and then remains on $I$;

(iv) if $x \in[0.11,0.8]$ then its orbit either converges to the pseudo equilibrium $(0,0)$, or converges to the stable focus $(0.8,-0.5129)$, or remains on $I$, or converges to $S$ in forward time. In backward time its orbit reaches the visible fold $(0.8,0)$ in a finite time and then remains on $I$.

\subsection{Displacement function theorem}

Our second main result of this paper is about the development of the displacement function for a class of discontinuous differential systems. We shall use this result to prove Theorem 1 .

We consider the following discontinuous differential system

$$
r^{\prime}(\theta)=\varepsilon S_{1}(\theta, r)+\varepsilon^{2} S_{2}(\theta, r)+\varepsilon^{3} T(\theta, r, \varepsilon),
$$

with

$$
\begin{gathered}
S_{i}(\theta, r)=\left\{\begin{array}{ll}
S_{i}^{-}(\theta, r) & \text { if }-\pi \leq \theta \leq 0, \\
S_{i}^{+}(\theta, r) & \text { if } 0 \leq \theta \leq \pi,
\end{array}\right. \text { and } \\
T(\theta, r, \varepsilon)= \begin{cases}T^{-}(\theta, r, \varepsilon) & \text { if }-\pi \leq \theta \leq 0, \\
T^{+}(\theta, r, \varepsilon) & \text { if } 0 \leq \theta \leq \pi,\end{cases}
\end{gathered}
$$

where $S_{i}^{-}:[-\pi, 0] \times\left[0, \rho^{*}\right] \rightarrow \mathbb{R}$ and $S_{i}^{+}:[0, \pi] \times\left[0, \rho^{*}\right] \rightarrow \mathbb{R}$ for $i=1,2$ and $T^{-}:[-\pi, 0] \times\left[0, \rho^{*}\right] \times\left[0, \varepsilon_{0}\right] \rightarrow \mathbb{R}$ and $T^{+}$: $[0, \pi] \times\left[0, \rho^{*}\right] \times\left[0, \varepsilon_{0}\right] \rightarrow \mathbb{R}$ are analytical functions $2 \pi$-periodic in the variable $\theta$. The discontinuous set of system (2) consists in the union of the two rays $\Sigma_{0}=\{\theta=0, \rho \geq 0\}$ and $\Sigma_{1}=\{\theta=\pi, \rho \geq 0\}$.

Let $r(\cdot, \rho, \varepsilon):[-\pi, \pi] \rightarrow \mathbb{R}$ be the solution of system (2) such that $r(0, \rho)=\rho$. Let $r^{+}(\cdot, \rho, \varepsilon):[0, \pi] \rightarrow \mathbb{R}$ and $r^{-}(\cdot, \rho, \varepsilon)$ : $[-\pi, 0] \rightarrow \mathbb{R}$ be the solutions of the systems

$$
r^{\prime}(\theta)=\varepsilon S_{1}^{ \pm}(\theta, r)+\varepsilon^{2} S_{2}^{ \pm}(\theta, r)+\varepsilon^{2} T^{ \pm}(\theta, r, \varepsilon),
$$

respectively, such that $r^{ \pm}(0, \rho, \varepsilon)=\rho$. Clearly

$$
r(\theta, \rho, \varepsilon)= \begin{cases}r^{-}(\theta, \rho, \varepsilon) & \text { if }-\pi \leq \theta \leq 0 \\ r^{+}(\theta, \rho, \varepsilon) & \text { if } 0 \leq \theta \leq \pi\end{cases}
$$

for more details see Fig. 5 .

Now let $\Delta:\left[0, \rho^{*}\right] \times\left[0, \varepsilon_{0}\right] \rightarrow \mathbb{R}$ be the displacement function defined as $\Delta(\rho, \varepsilon)=r^{+}(\pi, \rho, \varepsilon)-r^{-}(-\pi, \rho, \varepsilon)($ see again Fig. 5). Given $(\bar{\rho}, \bar{\varepsilon}) \in\left(0, \rho^{*}\right) \times\left(0, \varepsilon_{0}\right]$ it is easy to prove that the function $r(t, \bar{\rho}, \bar{\varepsilon})$ is a crossing periodic solution of system $(2)$, for $\varepsilon=\bar{\varepsilon}$, if and only if $\Delta(\bar{\rho}, \bar{\varepsilon})=0$. Moreover $r(t, \bar{\rho}, \bar{\varepsilon})$ is a crossing limit cycle of system (2), for $\varepsilon=\bar{\varepsilon}$, if and only if there exists a neighborhood $I$ of $\bar{\rho}$ such that $\Delta(\rho, \bar{\varepsilon}) \neq 0$ for every $\rho \in I \backslash\{\bar{\rho}\}$.

We define the functions $\Delta_{1}, \Delta_{2}:\left[0, \rho^{*}\right] \rightarrow \mathbb{R}$ as

$$
\Delta_{1}(\rho)=\int_{0}^{\pi}\left[S_{1}^{+}(\theta, \rho)+S_{1}^{-}(\theta-\pi, \rho)\right] d \theta, \quad \text { and }
$$




$$
\begin{aligned}
\Delta_{2}(\rho)= & \int_{0}^{\pi}\left[S_{2}^{+}(\theta, \rho)+S_{2}^{-}(\theta-\pi, \rho)\right] d \theta \\
& +\int_{0}^{\pi}\left[\frac{\partial}{\partial r} S_{1}^{+}(\theta, \rho) r_{1}^{+}(\theta, \rho)+\frac{\partial}{\partial r} S_{1}^{-}(\theta-\pi, \rho) r_{1}^{-}(\theta-\pi, \rho)\right] d \theta .
\end{aligned}
$$

Here the functions $r_{1}^{ \pm}:(-\pi, \pi) \times \mathbb{R}^{+} \rightarrow \mathbb{R}$ are defined as

$$
r_{1}^{ \pm}(\theta, r)=\int_{0}^{\theta} S_{1}^{ \pm}(\phi, r) d \phi .
$$

In order to estimate the zeros of the function $\Delta(\rho, \varepsilon)$ we shall write it in terms of the functions $\Delta_{1}(\rho)$ and $\Delta_{2}(\rho)$. It is stated in the next theorem.

Theorem 2. For system (2) the equality $\Delta(\rho, \varepsilon)=\varepsilon \Delta_{1}(\rho)+\varepsilon^{2} \Delta_{2}(\rho)+\mathcal{O}\left(\varepsilon^{3}\right)$ holds for $\varepsilon>0$ sufficiently small.

The notation $\mathcal{O}$ has been used in the statement of Theorem 2. It is one of the Landau's symbols which we clarify in the sequel. Let $g:\left(-\delta_{0}, \delta_{0}\right) \rightarrow \mathbb{R}$ be a function defined on the small interval $\left(-\delta_{0}, \delta_{0}\right)$. We say that $g(\delta)=\mathcal{O}\left(\delta^{\ell}\right)$ for some positive integer $\ell$ if there exist constants $\delta_{1}>0$ and $\Lambda>0$ such that $|g(\delta)| \leq \Lambda\left|\delta^{\ell}\right|$ for $-\delta_{1} \leq \delta \leq \delta_{1}$. The symbol $\mathcal{O}$ is one of the Landau's symbols (see for instance [19]).

\section{Preliminary results}

In this section, following [8], we present the basic notations and results on the Bendixson transformation that we shall use for proving Theorem 1 in Section 3.

The Bendixson transformation

$$
\left(\begin{array}{l}
u \\
v
\end{array}\right)=\frac{1}{x^{2}+y^{2}}\left(\begin{array}{l}
x \\
y
\end{array}\right),
$$

reduces the study of the dynamics in a neighborhood of infinity for planar vector fields to study the dynamics of the transformed system in a neighborhood of the origin, see for instance Andronov et al. [1].

Let $f$ and $g$ be Lipschitz functions in the variables $(x, y)$ and $\varepsilon>0$ a small parameter. The following planar system is considered

$$
\begin{gathered}
\dot{x}=f(x, y, \varepsilon), \\
\dot{y}=g(x, y, \varepsilon) .
\end{gathered}
$$

We obtain, through the Bendixson change of variables (5), an equivalent system which behaves in a neighborhood of the origin like system (6) near infinity. Composing now the Bendixson change of variables (5) with the polar $\operatorname{coordinates} u=r \cos \theta$, $v=r \sin \theta$, system (6) becomes

$$
\begin{aligned}
& \dot{r}=R(r, \theta, \varepsilon)=-r^{2}\left[f\left(\frac{\cos \theta}{r}, \frac{\sin \theta}{r}, \varepsilon\right) \cos \theta+g\left(\frac{\cos \theta}{r}, \frac{\sin \theta}{r}, \varepsilon\right) \sin \theta\right], \\
& \dot{\theta}=\Theta(r, \theta, \varepsilon)=-r\left[f\left(\frac{\cos \theta}{r}, \frac{\sin \theta}{r}, \varepsilon\right) \cos \theta-g\left(\frac{\cos \theta}{r}, \frac{\sin \theta}{r}, \varepsilon\right) \cos \theta\right] .
\end{aligned}
$$

The Bendixson transformation composed with the polar coordinates gives rise to the following change of variables

$$
x=\frac{\cos \theta}{r}, \quad y=\frac{\sin \theta}{r},
$$

which we shall call by the polar Bendixson transformation.

We shall study the flow of system (7) contained in the half-cylinder $\mathbb{R}^{+} \times \mathbb{S}^{1}=\{(r, \theta): r \geq 0, \theta \in[-\pi, \pi)\}$. So system (7) has to be well defined for $r=0$ which does not happens in most cases. Nevertheless with a time reparametrization, usually multiplying the vector field by an adequate power of $r$, we can overcome this difficulty, thus we shall assume that system (7) is well defined for $r=0$. We note that in this case the existence of a periodic orbit at infinity for system (6) is equivalent to have $r=0$ as a periodic orbit on the cylinder for system (7). In the sequel we assume that

(A1) the functions $R$ and $\Theta$ are Lipschitz functions in the variable $r$ and they have period $2 \pi$ in the variable $\theta$.

(A2) $R(0, \theta, \varepsilon)=0$ and $\Theta(0, \theta, \varepsilon) \neq 0$ for all $\theta \in \mathbb{S}^{1}$ and for every $\varepsilon \geq 0$ sufficiently small.

Note that the last assumption gives a sufficient and necessary condition in order that system (6) has a periodic orbit at infinity. Indeed for $\varepsilon \geq 0$ sufficiently small $r=0$ is a periodic orbit of system (7), moreover it has no equilibrium points in [0, $\left.\rho^{*}\right] \times \mathbb{S}^{1}$ for some $\rho^{*}>0$ sufficiently small.

The differential system (7) can be driven into the first order differential equation

$$
r^{\prime}=\frac{d r}{d \theta}=S(r, \theta, \varepsilon)=\frac{R(r, \theta, \varepsilon)}{\Theta(r, \theta, \varepsilon)},
$$

where $r \in\left[0, \rho^{*}\right], \theta \in \mathbb{S}^{1}$, and $\varepsilon \geq 0$ is a small parameter, because $\Theta \neq 0$ in a neighborhood of $r=0$ for $\varepsilon \geq 0$ sufficiently small. 


\section{Proofs of Theorems 1 and 2}

In the proof of Theorem 1 we shall use the following lemma.

Lemma 3. For $a, b, c$, and d real numbers, let $f(\rho, \varepsilon)=a \rho^{2}+\varepsilon\left(b \rho^{3}+c \rho^{2}+d \rho\right)+\mathcal{O}\left(\varepsilon^{2}\right)$ be a $\mathcal{C}^{1}$ function. If $f(0$, $\varepsilon)=0$ for $\varepsilon \geq 0$ sufficiently small and $a \cdot d<0$, then there exist $\varepsilon_{0}>0$ and a unique $\mathcal{C}^{1}$ function $\rho(\varepsilon)$ such that $\rho(0)=0, \rho(\varepsilon)>0$ and $f(\rho(\varepsilon), \varepsilon)=0$ for all $\varepsilon \in\left(0, \varepsilon_{0}\right]$. Moreover for each $\varepsilon \in\left(0, \varepsilon_{0}\right], \rho(\varepsilon)$ is an isolated zero of $f(\cdot, \varepsilon)$ such that $(\partial f / \partial \rho)(\rho(\varepsilon), \varepsilon) \cdot a>0$.

Proof. We note that there exists a $C^{1}$ function $\xi(\rho, \varepsilon)$ such that $f(\rho, \varepsilon)=a \rho^{2}+\varepsilon\left(b \rho^{3}+c \rho^{2}+d \rho\right)+\varepsilon^{2} \xi(\rho, \varepsilon)$. Since $f(0, \varepsilon)=$ 0 for $\varepsilon \geq 0$ sufficiently small we have that $\xi(0, \varepsilon)=0$ for $\varepsilon \geq 0$ sufficiently small. We note that every $\mathcal{C}^{1}$ branch $\rho(\varepsilon)$ of zeros of the function $f(\rho, \varepsilon)$ must satisfies $\rho(\varepsilon)=\varepsilon \zeta(\varepsilon)$ for some $\mathcal{C}^{1}$ function $\zeta(\varepsilon)$. So taking $\rho=\varepsilon \zeta$ we consider the function

$$
g(\zeta, \varepsilon)=\frac{f(\varepsilon \zeta, \varepsilon)}{\varepsilon^{2}}=a \zeta^{2}+d \zeta+\varepsilon c \zeta^{2}+\varepsilon^{2} b \zeta^{3}+\xi(\varepsilon \zeta, \varepsilon)
$$

Since $\xi(\varepsilon \zeta, \varepsilon)=\xi(0,0)+\mathcal{O}(\varepsilon)$ and $\xi(0,0)=0$ we conclude that $g(\zeta, \varepsilon)=a \zeta^{2}+d \zeta+\mathcal{O}(\varepsilon)$. The equation $g(\zeta, 0)=0$ has two solutions, namely $\zeta=0$ and $\zeta=\zeta^{*}=-d / a>0$. Moreover $(\partial g / \partial \zeta)(0,0)=d \neq 0$ and $(\partial g / \partial \zeta)\left(\zeta^{*}, 0\right)=-d \neq 0$. From the Implicit Function Theorem we conclude that there exists $\varepsilon_{0}>0$, a unique $\mathcal{C}^{1}$ function $\zeta_{0}(\varepsilon)$ such that $\zeta_{0}(0)=0$ and $g\left(\zeta_{0}(\varepsilon), \varepsilon\right)=0$ for every $\varepsilon \in\left(0, \varepsilon_{0}\right]$, and a unique $\mathcal{C}^{1}$ function $\zeta(\varepsilon)$ such that $\zeta(0)=\zeta^{*}, \zeta(\varepsilon)>0$ and $g(\zeta(\varepsilon), \varepsilon)=0$ for every $\varepsilon \in\left(0, \varepsilon_{0}\right]$. Since $g(0, \varepsilon)=0$ we obtain, from the uniqueness of $\zeta_{0}$, that $\zeta_{0}=0$. Hence taking $\rho(\varepsilon)=\varepsilon(\zeta(\varepsilon))$ we have that $\rho(0)=0, \rho(\varepsilon)>0$ and $f(\rho(\varepsilon), \varepsilon)=0$ for every $\varepsilon \in\left(0, \varepsilon_{0}\right]$. Moreover $(\partial f / \partial \rho)(\rho(\varepsilon), \varepsilon) \cdot a=-\varepsilon^{2} a d+\mathcal{O}\left(\varepsilon^{3}\right)$, so taking $\varepsilon_{0}$ small enough we obtain that $(\partial f / \partial \rho)(\rho(\varepsilon), \varepsilon) \cdot a>0$ for every $\varepsilon \in\left(0, \varepsilon_{0}\right]$. It completes this proof.

Now we prove Theorem 1.

Proof of Theorem 1. Applying the polar Bendixson transformation to system (1) we compute $(R, \Theta)=\left(R^{+}, \Theta^{+}\right)$for $y>0$, and $(R, \Theta)=\left(R^{-}, \Theta^{-}\right)$for $y<0$, as

$$
\begin{aligned}
R^{ \pm}(0, \theta, \varepsilon)= & \varepsilon\left(r^{2}\left(-a^{ \pm} \cos \theta-k^{ \pm} \sin \theta\right)+\frac{1}{2} r\left(-\left(c^{ \pm}+m^{ \pm}\right) \sin (2 \theta)+\left(n^{ \pm}-b^{ \pm}\right) \cos (2 \theta)-b^{ \pm}-n^{ \pm}\right)\right) \\
& +\varepsilon^{2}\left(r^{2}\left(-\alpha^{ \pm} \cos \theta-\kappa^{ \pm} \sin \theta\right)+\frac{1}{2} r\left(-\left(\gamma^{ \pm}+\mu^{ \pm}\right) \sin (2 \theta)+\left(v^{ \pm}-\beta^{ \pm}\right) \cos (2 \theta)-\beta^{ \pm}-v^{ \pm}\right)\right), \quad \text { and } \\
\Theta^{ \pm}(0, \theta, \varepsilon)= & 1+\varepsilon \cos \theta\left(r\left(k^{ \pm}-a^{ \pm} \tan \theta\right)-\sin \theta\left(c^{ \pm} \tan \theta+b^{ \pm}-n^{ \pm}\right)\right. \\
& \left.+m^{ \pm} \cos \theta\right)+\varepsilon^{2} \cos \theta\left(r\left(\kappa^{ \pm}-\alpha^{ \pm} \tan \theta\right)-\sin \theta\left(\gamma^{ \pm} \tan \theta+\beta^{ \pm}-v^{ \pm}\right)+\mu^{ \pm} \cos \theta\right)
\end{aligned}
$$

It is easy to see that the assumptions $(A 1)$ and $(A 2)$ hold for the functions $R^{ \pm}, \Theta^{ \pm}$. Thus $r=0$ is a periodic solution for both systems $\dot{r}=S^{+}(\theta, r, \varepsilon)$ and $\dot{r}=S^{-}(\theta, r, \varepsilon)$, so $r=0$ is also a periodic solution of the discontinuous piecewise system

$$
\dot{r}= \begin{cases}S^{-}(\theta, r, \varepsilon) & \text { if }-\pi \leq \theta \leq 0, \\ S^{+}(\theta, r, \varepsilon) & \text { if } 0 \leq \theta \leq \pi,\end{cases}
$$

which is equivalent to system (1).

Computing now the Taylor series of $S^{ \pm}(\theta, r, \varepsilon)$ near $\varepsilon=0$ we have that system (1) is equivalent to system (2) by taking

$$
\begin{aligned}
S_{1}^{ \pm}(\theta, r)= & -r\left(b^{ \pm} \cos ^{2} \theta+\left(c^{ \pm}+m^{ \pm}\right) \cos \theta \sin \theta+n^{ \pm} \sin ^{2} \theta\right)-r^{2}\left(a^{ \pm} \cos \theta+k^{ \pm} \sin \theta\right), \\
S_{2}^{ \pm}(\theta, r)= & r \sec ^{2} \theta\left(\operatorname { c o s } ^ { 4 } \theta ( r ( k ^ { \pm } - a ^ { \pm } \operatorname { t a n } \theta ) - \operatorname { s i n } \theta ( c ^ { \pm } \operatorname { t a n } \theta + b ^ { \pm } - n ^ { \pm } ) + m ^ { \pm } \operatorname { c o s } \theta ) \left(r\left(a^{ \pm}+k^{ \pm} \tan \theta\right)\right.\right. \\
& \left.+\sin \theta\left(c^{ \pm}+n^{ \pm} \tan \theta+m^{ \pm}\right)+b^{ \pm} \cos \theta\right)-\cos ^{2} \theta\left(\cos \theta\left(\alpha^{ \pm} r+\left(\gamma^{ \pm}+\mu^{ \pm}\right) \sin \theta\right)+\beta^{ \pm} \cos ^{2} \theta\right. \\
& \left.\left.+\sin \theta\left(\kappa^{ \pm} r+v^{ \pm} \sin \theta\right)\right)\right) .
\end{aligned}
$$

On one hand, the Bendixon polar transformation allows us to study all the crossing limit cycles of system (1) coming from the infinity. On the other hand, the crossing limit cycles coming from the origin are studied using the usual polar coordinates transformation $(x, y)=(r \cos \theta, r \sin \theta)$. The birth of crossing limit cycles from some finite periodic solutions of the linear center (that is, system $(1)$ when $\varepsilon=0$ ) is studied through both transformations. We then repeat the above procedure using now the polar change of variables to obtain a transformed system similar to system (9) (we shall omit these standard computations and expressions).

In order to avoid any misunderstanding we denote by $\Delta^{B}(\varepsilon, \rho)$ the displacement function related to the transformed system (9) (via polar Bendixson transformation), and we denote by $\Delta^{P}(\varepsilon, \rho)$ the displacement function related to system (1) transformed via polar change of variables. We define $f^{B}(\rho, \varepsilon)=\Delta^{B}(\varepsilon, \rho) / \varepsilon$ and $f^{P}(\rho, \varepsilon)=\Delta^{P}(\varepsilon, \rho) / \varepsilon$.

Firstly, assuming $\left(b^{+}+b^{-}+n^{+}+n^{-}\right)\left(k^{-}-k^{+}\right) \neq 0$ we compute

$$
\begin{aligned}
& \Delta_{1}^{B}(\rho)=2\left(k^{-}-k^{+}\right) \rho^{2}-\frac{1}{2}\left(b^{+}+b^{-}+n^{+}+n^{-}\right) \pi \rho, \\
& \Delta_{1}^{P}(\rho)=\frac{1}{2}\left(b^{+}+b^{-}+n^{+}+n^{-}\right) \pi \rho-2\left(k^{-}-k^{+}\right) .
\end{aligned}
$$


So, from Theorem $2, f^{B}(\rho, \varepsilon)=\Delta_{1}^{B}(\rho)+\mathcal{O}(\varepsilon)$ and $f^{P}(\rho, \varepsilon)=\Delta_{1}^{P}(\rho)+\mathcal{O}(\varepsilon)$. From the hypotheses of statement $(i)$ we have that $\rho_{1}^{*}=4\left(k^{-}-k^{+}\right) /\left(\left(b^{+}+b^{-}+n^{+}+n^{-}\right) \pi\right)>0, f^{P}\left(\rho_{1}^{*}, 0\right)=0$ and $\left(\partial f^{P} / \partial \rho\right)\left(\rho_{1}^{*}, 0\right)=\left(b^{+}+b^{-}+n^{+}+n^{-}\right) \pi / 2 \neq 0$. Hence, using the Implicit Function Theorem, we conclude that, for $|\varepsilon| \neq 0$ sufficiently small, there exists a unique $\mathcal{C}^{1}$ function $\rho_{1}(\varepsilon)$ such that $f^{P}\left(\rho_{1}(\varepsilon), \varepsilon\right)=0$ and $\rho_{1}(\varepsilon) \rightarrow \rho_{1}^{*}$. It leads to the existence of a unique crossing limit cycle $\left(x_{1}(t, \varepsilon), y_{1}(t, \varepsilon)\right)$, of system $(1)$, that comes from a finite periodic solution of the linear center, namely $\left|\left(x_{1}(0, \varepsilon), y_{1}(0, \varepsilon)\right)\right|=\rho_{1}(\varepsilon) \rightarrow \rho_{1}^{*}$ when $\varepsilon \rightarrow 0$. Moreover, this limit cycle is stable (resp. unstable) provided that $b^{+}+b^{-}+n^{+}+n^{-}<0$ (resp. $b^{+}+b^{-}+n^{+}+n^{-}<0$ ). From the expressions of $\Delta_{1}^{P}(\rho)$ and hypotheses of statement $(i)$, there are no limit cycles coming from the origin. Now, since $r=0$ is a periodic solution of system (9) (that is, the infinity of system (1)) we have that $f^{B}(0, \varepsilon)=0$ for every $|\varepsilon| \neq 0$ small enough. Furthermore, the equation $f^{B}(\rho, 0)=0$ admits two zeros, namely $\rho=0$ and $\rho=1 / \rho_{1}^{*}$. Since $\left(\partial f^{B} / \partial \rho\right)(0,0)=-\pi\left(b^{+}+b^{-}+n^{+}+n^{-}\right) / 2 \neq 0$ we conclude, using a similar argument that we have used to prove Lemma 3, that this zero corresponds only to the infinity. Therefore we conclude that there are no limit cycles coming from the infinity, which leads us to the uniqueness of the crossing limit cycle found above. It concludes the proof of statement $(i)$.

Secondly, assuming $k^{-}=k^{+}$and $b^{+}+b^{-}+n^{+}+n^{-} \neq 0$ we compute

$$
\begin{aligned}
& \Delta_{1}^{P}(\rho)=-\Delta_{1}^{B}(\rho)=\frac{1}{2}\left(b^{+}+b^{-}+n^{+}+n^{-}\right) \pi \rho, \\
& \Delta_{2}^{P}(\rho)=\lambda_{1} \rho+\lambda_{2},
\end{aligned}
$$

where

$$
\begin{aligned}
\lambda_{1}= & \frac{1}{8} \pi\left(2\left(c^{-}-m^{-}\right)\left(b^{-}+n^{-}\right)-\left(b^{-}-b^{+}+n^{-}-n^{+}\right)\left(b^{-}+b^{+}+n^{-}+n^{+}\right) \pi\right. \\
& \left.+2\left(c^{+}-m^{+}\right)\left(b^{+}+n^{+}\right)+4\left(\beta^{-}+\beta^{+}+v^{-}+v^{+}\right)\right), \\
\lambda_{2}= & 2\left(k^{+}\left(m^{-}-m^{+}\right)+a^{+}\left(b^{+}+n^{+}\right)+\kappa^{+}-\kappa^{-}\right)-2 a^{-}\left(b^{-}+n^{-}\right),+\frac{1}{2} k^{+}\left(b^{+}+b^{-}+n^{+}+n^{-}\right) \pi .
\end{aligned}
$$

So, from Theorem 2, $f^{B}(\rho, \varepsilon)=\Delta_{1}^{B}(\rho)+\mathcal{O}(\varepsilon)$ and $f^{P}(\rho, \varepsilon)=\Delta_{1}^{P}(\rho)+\varepsilon \Delta_{2}^{P}(\rho)+\mathcal{O}\left(\varepsilon^{2}\right)$. The equation $f^{B}(\rho, 0)=0$ admits only one zero $\rho=0$. Since $\left(\partial f^{B} / \partial \rho\right)(0,0)=-\pi\left(b^{+}+b^{-}+n^{+}+n^{-}\right) / 2 \neq 0$ we conclude again that this zero corresponds only to the infinity, which is a periodic solutions of system (1). Therefore we conclude that there are no crossing limit cycles, of system (1), coming nether from the infinity nor from the finite periodic solutions of the linear center. Now we define $g^{P}(\zeta, \varepsilon)=f^{P}(\varepsilon \zeta, \varepsilon) / \varepsilon=\left(b^{+}+b^{-}+n^{+}+n^{-}\right) \pi \rho / 2+\lambda_{2}+\mathcal{O}(\varepsilon)$. From the hypotheses of statement (ii) we have that $\zeta_{2}^{*}=-2 \lambda_{2} /\left(\left(b^{+}+b^{-}+n^{+}+n^{-}\right) \pi\right)>0, g^{P}\left(\zeta_{2}^{*}, 0\right)=0$ and $\left(\partial g^{P} / \partial \rho\right)\left(\zeta_{2}^{*}, 0\right)=\left(b^{+}+b^{-}+n^{+}+n^{-}\right) \pi / 2 \neq 0$. Hence, using the Implicit Function Theorem, we conclude that, for $\varepsilon>0$ sufficiently small, there exists a unique $\mathcal{C}^{1}$ function $\zeta_{2}(\varepsilon)$ such that $f^{P}\left(\varepsilon \zeta_{2}(\varepsilon), \varepsilon\right)=g^{P}\left(\zeta_{2}(\varepsilon), \varepsilon\right)=0$ and $\zeta_{2}(\varepsilon) \rightarrow \zeta_{2}^{*}$. It leads to the existence of a unique crossing limit cycle $\left(x_{2}(t, \varepsilon), y_{2}(t, \varepsilon)\right)$, of system (1), that comes from the origin, indeed $\left|\left(x_{2}(0, \varepsilon), y_{2}(0, \varepsilon)\right)\right|=\varepsilon \zeta(\varepsilon) \rightarrow 0$ when $\varepsilon \rightarrow 0$. Moreover, this limit cycle is stable (resp. unstable) provided that $b^{+}+b^{-}+n^{+}+n^{-}<0$ (resp. $b^{+}+b^{-}+n^{+}+n^{-}<0$ ). It concludes the proof of statement (ii).

Finally, we assume that $b^{+}+b^{-}+n^{+}+n^{-}=0$. Since $r=0$ is a periodic solution of system $(9)$ we have that $f^{B}(0, \varepsilon)=$ $\Delta^{B}(0, \varepsilon) / \varepsilon=0$ for every $\varepsilon>0$ small enough. Applying Theorem 2 we compute $f^{B}(\varepsilon, \rho)=\Delta_{1}^{B}(\rho)+\varepsilon \Delta_{2}^{B}(\rho)+\mathcal{O}\left(\varepsilon^{2}\right)$ and $f^{P}(\varepsilon, \rho)=\Delta_{1}^{P}(\rho)+\mathcal{O}(\varepsilon)$, with $\Delta_{1}^{B}(\rho)=a \rho^{2}, \Delta_{2}^{B}(\rho)=b \rho^{3}+c \rho^{2}+d \rho$, and $\Delta_{1}^{P}(\rho)=-a$, where

$$
\begin{aligned}
& a=2\left(k^{-}-k^{+}\right), \\
& b=4\left(k^{+}+k^{-}\right)\left(k^{+}-k^{-}\right), \\
& c=\frac{1}{2}\left(b^{+}+n^{+}\right)\left(3 \pi\left(k^{+}-k^{-}\right)-4\left(a^{+}+a^{-}\right)\right)+2\left(k^{+} m^{+}-k^{-} m^{-}+\kappa^{-}-\kappa^{+}\right), \\
& d=\frac{\pi}{4}\left(\left(b^{+}+n^{+}\right)\left(c^{-}-c^{+}+m^{+}-m^{-}\right)-2\left(\beta^{+}+\beta^{+}+v^{+}+v^{-}\right)\right) .
\end{aligned}
$$

From hypotheses of statement (iii) we get

$$
a \cdot d=\frac{\pi}{2}\left(k^{-}-k^{+}\right)\left(\left(b^{+}+n^{+}\right)\left(c^{-}-c^{+}+m^{+}-m^{-}\right)-2\left(\beta^{+}+\beta^{+}+v^{+}+v^{-}\right)\right)<0 .
$$

Hence using Lemma 3 we conclude that, for $\varepsilon>0$ sufficiently small, there exists a unique $\mathcal{C}^{1}$ function $\rho_{3}(\varepsilon)$ such that $\rho_{3}(0)=0$, $\rho_{3}(\varepsilon)>0$ and $f^{B}\left(\rho_{3}(\varepsilon), \varepsilon\right)=0$. It leads to the existence of a unique crossing limit cycle $\left(x_{3}(t, \varepsilon), y_{3}(t, \varepsilon)\right)$, of system (1), that comes from the infinity, indeed $\left|\left(x_{3}(0, \varepsilon), y_{3}(0, \varepsilon)\right)\right|=1 / \rho_{3}(\varepsilon) \rightarrow \infty$ when $\varepsilon \rightarrow 0$. Also from Lemma 3 this limit cycle is stable (resp. unstable) provided that $a=2\left(k^{-}-k^{+}\right)<0$ (resp. $\left.a=2\left(k^{-}-k^{+}\right)>0\right)$. Now, since $f^{P}(\rho, \varepsilon)=2\left(k^{+}-k^{-}\right)+\mathcal{O}(\varepsilon)$ we conclude that there are no crossing limit cycles, of system (1), coming neither from the origin nor from the finite periodic solutions of the linear center. It completes the proof of statement (iii) and, consequently, the proof of this theorem.

The proof of Theorem 2 follows the ideas contained in [11].

Proof of Theorem 2. By continuity of the solution $r(\theta, \rho, \varepsilon)$ of system (2) and by compactness of the set [- $\pi, \pi] \times\left[0, \rho^{*}\right] \times$ $\left[0, \varepsilon_{0}\right]$, there exists a compact subset $K$ of $[0, \infty)$ such that $r(\theta, \rho, \varepsilon) \in K$ for all $\theta \in[-\pi, \pi], \rho \in\left[0, \rho^{*}\right]$ and $\varepsilon \in\left[0, \varepsilon_{0}\right]$. Now by the continuity of the function $T,|T(\phi, r(\phi, \rho, \varepsilon), \varepsilon)| \leq \max \left\{|T(\theta, \rho, \varepsilon)|:(\theta, \rho, \varepsilon) \in[-\pi, \pi] \times\left[0, \rho^{*}\right] \times\left[0, \varepsilon_{0}\right]\right\}=M$. Then

$$
\left|\int_{-\pi}^{\theta} T(\phi, r(\phi, \rho, \varepsilon), \varepsilon) d \phi\right| \leq \int_{-\pi}^{\pi}|T(\phi, r(\phi, \rho, \varepsilon), \varepsilon)| d \phi \leq 2 \pi M
$$


which implies that $\int_{-\pi}^{\theta} T(\phi, r(\phi, \rho, \varepsilon), \varepsilon) d \phi=\mathcal{O}(1)$. So

$$
r^{ \pm}(\theta, \rho, \varepsilon)=\rho+\varepsilon \int_{0}^{\theta} S_{1}^{ \pm}\left(\phi, r^{ \pm}(\phi, \rho, \varepsilon)\right) d \phi+\varepsilon^{2} \int_{0}^{\theta} S_{2}^{ \pm}\left(\phi, r^{+}(\phi, \rho, \varepsilon), \varepsilon\right) d \phi+\mathcal{O}\left(\varepsilon^{3}\right) .
$$

Expanding the functions $\varepsilon \mapsto S_{i}^{ \pm}\left(\phi, r^{ \pm}(\phi, \rho, \varepsilon)\right)$ around $\varepsilon=0$ for $i=1,2$ we have

$$
r^{ \pm}(\theta, \rho, \varepsilon)=\rho+\varepsilon \int_{0}^{\theta} S_{1}^{ \pm}(\phi, \rho) d \phi+\varepsilon^{2} \int_{0}^{\theta}\left[\frac{\partial}{\partial r} S_{1}^{ \pm}(\phi, \rho) r_{1}^{ \pm}(\phi, r)+S_{2}^{ \pm}(\phi, \rho)\right] d \phi+\mathcal{O}\left(\varepsilon^{3}\right),
$$

because

$$
\left.\frac{\partial}{\partial \varepsilon} r^{ \pm}(\theta, \rho, \varepsilon)\right|_{\varepsilon=0}=\int_{0}^{\theta} S_{1}^{ \pm}(\phi, \rho) d \phi=r_{1}^{ \pm}(\theta, r) .
$$

So we compute

$$
\begin{aligned}
\Delta(\rho, \varepsilon)= & r^{+}(\pi, \rho, \varepsilon)-r^{-}(-\pi, \rho, \varepsilon) \\
= & \varepsilon\left(\int_{0}^{\pi} S_{1}^{+}(\theta, \rho) d \theta-\int_{0}^{-\pi} S_{1}^{-}(\theta, \rho) d \theta\right)+\varepsilon^{2}\left(\int_{0}^{\pi}\left[\frac{\partial}{\partial r} S_{1}^{+}(\theta, \rho) r_{1}^{+}(\theta, r)+S_{2}^{+}(\theta, \rho)\right] d \theta\right. \\
& \left.-\int_{0}^{-\pi}\left[\frac{\partial}{\partial r} S_{1}^{-}(\theta, \rho) r_{1}^{-}(\theta, r)+S_{2}^{-}(\theta, \rho)\right] d \theta\right)+\mathcal{O}\left(\varepsilon^{3}\right) \\
= & \varepsilon\left(\int_{0}^{\pi} S_{1}^{+}(\theta, \rho) d \theta+\int_{-\pi}^{0} S_{1}^{-}(\theta, \rho) d \theta\right)+\varepsilon^{2}\left(\int_{0}^{\pi}\left[\frac{\partial}{\partial r} S_{1}^{+}(\theta, \rho) r_{1}^{+}(\theta, r)+S_{2}^{+}(\theta, \rho)\right] d \theta\right. \\
& \left.+\int_{-\pi}^{0}\left[\frac{\partial}{\partial r} S_{1}^{-}(\theta, \rho) r_{1}^{-}(\theta, r)+S_{2}^{-}(\theta, \rho)\right] d \theta\right)+\mathcal{O}\left(\varepsilon^{3}\right) \\
= & \varepsilon\left(\int_{0}^{\pi}\left[S_{1}^{+}(\theta, \rho)+S_{1}^{-}(\theta-\pi, \rho)\right] d \theta\right) \\
& +\varepsilon^{2}\left(\int _ { 0 } ^ { \pi } \left[S_{2}^{+}(\theta, \rho)+S_{2}^{-}(\theta-\pi, \rho)+\frac{\partial}{\partial r} S_{1}^{+}(\theta, \rho) r_{1}^{+}(\theta, r)\right.\right. \\
& \left.\left.+\frac{\partial}{\partial r} S_{1}^{-}(\theta-\pi, \rho) r_{1}^{-}(\theta-\pi, r)\right] d \theta\right)+\mathcal{O}\left(\varepsilon^{3}\right) \\
= & \varepsilon \Delta_{1}(\rho)+\varepsilon^{2} \Delta_{2}(\rho)+\mathcal{O}\left(\varepsilon^{3}\right) .
\end{aligned}
$$

It completes the proof of Theorem 2 .

\section{Acknowledgments}

We thank to the referees for their helpful comments and suggestions.

The first author is partially supported by a PROCAD-CAPES grant 88881.068 462/2014-01 and by a FAPESP grant 2013/13344-0. The second author is partially supported by a MINECO/FEDER grant MTM2008-03437, MINECO grant MTM2013-40998-P, an AGAUR grant number 2014SGR-568, an ICREA Academia, the grants FP7-PEOPLE-2012-IRSES 318999 and 316338, the MINECO/FEDER grant UNAB13-4E-1604. The third author is partially supported by a FAPESP grant 2012/10231-7. The three authors are also supported by a CAPES CSF-PVE grant 88881.030454/2013-01 from the program CSF-PVE.

\section{References}

[1] A.A. Andronov, E.A. Leontovich, I.I. Gordon, A.G. Maier, Qualitative Theory of Second-Order Dynamic Systems, John Wiley and Sons, New York, Toronto, 1973.

[2] A.A. Andronov, A.A. Vitt, S.E. Khaikin, Theory of Oscillators, International Series of Monographs in Physics, 4, Pergamon Press, 1966.

[3] C. Buzzi, C. Pessoa, J. Torregrosa, Piecewise linear perturbations of a linear center, Discrete Continuous Dyn. Syst. 33 (2013) $3915-3936$.

[4] C. Christopher, C. Li, Limit cycles of differential equations, Advanced Courses in Mathematics CRM Barcelona, Birkhäuser Verlag, Basel, 2007.

[5] F. Dumortier, J. Llibre, J.C. Artés, Qualitative Theory of Planar Differential Systems, Universitext, Springer-Verlag, Berlin, 2006.

[6] A.F. Filippov, Differential equations with discontinuous righthand side, Mathematics and Its Applications, Kluwer Academic Publishers, Dordrecht, 1988.

[7] W. Huang, Y. Liu, W. Zhang, Bifurcation of limit cycles and isochronous center at infinity for a class of differential systems, J. Appl. Anal. Comput. 1 (2011) $397-410$.

[8] J. Llibre, E. Ponce, Hopf bifurcation from infinity for planar control system, Publicacions Matemàtiques 41 (1997) 181-198.

[9] J. Llibre, A.C. Mereu, Limit cycles for discontinuous quadratic differential systems with two zones, J. Math. Anal. Appl. 413 (2) (2014) $763-775$.

[10] J. Llibre, A.C. Mereu, D.D. Novaes, Averaging theory for discontinuous piecewise differential systems, J. Differ. Equ. 258 (2015) $4007-4032$.

[11] J. Llibre, D.D. Novaes, M.A. Teixeira, Higher order averaging theorem for finding periodic solutions via Brouwer degree, Nonlinearity 27 (2014) $563-583$.

[12] J. Llibre, D.D. Novaes, M.A. Teixeira, On the birth of limit cycles for non-smooth dynamical systems, Bull. Sci. Math. 139 (2015) $229-244$.

[13] J. Llibre, D.D. Novaes, M.A. Teixeira, Limit cycles bifurcating from the periodic orbits of a discontinuous piecewise linear differential center with two zones, Int. J. Bifurcation Chaos (2015), to appear. 
[14] J. Llibre, M.A. Teixeira, Limit cycles for m-piecewise discontinuous polynomial Liénard differential equations, Z. Angew. Math. Phys. 66 (2015) 51-66.

[15] O. Makarenkov, J.S.W. Lamb, Dynamics and bifurcation of nonsmooth systems: a survey, Physica D 241 (2012) 1826-1844.

[16] N. Minorski, Nonlinear Oscillations, Van Nostrand, New York, 1962.

[17] D.D. Novaes, On nonsmooth perturbations of nondegenerate planar centers, Publicacions Matemàtiques vol. Extra (2014) 395-420.

[18] D.D. Novaes, H. Ponce, A simple solution to the Braga-Mello conjecture, Int. J. Bifurcation Chaos 25 (2015) 1550009.

[19] J.A. Sanders, F. Verhulst, J. Murdock, Averaging Methods in Nonlinear Dynamical Systems, Applied Mathematical Sciences, 59, second ed., Springer, New York, 2007.

[20] Various, Special issue on dynamics and bifurcations of nonsmooth systems, Physica D 241 (22) (2012) 1825-2082.

[21] Q. Zhang, Y. Liu, A cubic polynomial system with seven limit cycles at infinity, Appl. Math. Comput. 177 (2006) 319-329.

[22] L. Zhang, Y. Liu, X. Jiang, The center conditions and bifurcation of limit cycles at the infinity for a cubic polynomial system, Appl. Math. Comput. 218 (2011) 1360-1370. 